HNO 2019 $67: 652-653$

https://doi.org/10.1007/s00106-019-0718-3

(c) Springer Medizin Verlag GmbH, ein Teil von Springer Nature 2019

\author{
C. Heiser ${ }^{1} \cdot$ T. Verse $^{2,3}$ \\ ' Klinik und Poliklinik für Hals-, Nasen- und Ohrenheilkunde, Klinikum rechts der Isar, Technische \\ Universität München, München, Deutschland \\ ${ }^{2}$ Klinik für Atemwegs-, Lungen- und Thoraxmedizin, Abteilung für HNO-Heilkunde, Kopf- und \\ Halschirurgie, Asklepios Klinikum Harburg, Hamburg, Deutschland \\ ${ }^{3}$ Medizinische Fakultät, Semmelweis Universität (Budapest), Asklepios Campus Hamburg, Hamburg, \\ Deutschland
}

\title{
Neue diagnostische und therapeutische Ansätze bei obstruktiver Schlafapnoe
}

Liebe Leserinnen und Leser,

in zuletztjährlichem Rhythmus findet der Kongress der International Sleep Surgical Society (ISSS) statt. Die Gesellschaft vereint Kollegen aus aller Welt, die sich mit der operativen Therapie von Schlafstörungen, allen voran der obstruktiven Schlafapnoe, beschäftigen. Längst hat sie es geschafft, alle führenden Kollegen zusammenzubringen. Der mit mittlerweile über 500 Teilnehmern weltweit größte internationale Kongress auf diesem Gebiet ist der wichtigste Impulsgeber für die Diagnostik und operative Therapie von Patienten mit schlafbezogenen Atmungsstörungen. Der Bedarf an Therapiealternativen für die Behandlung der obstruktiven Schlafapnoe (OSA) ist in den letzten Jahren mit zunehmender Prävalenz und „awareness“ gestiegen.

\section{》) Millionen von Menschen leiden unter der obstruktiven Schlafapnoe}

Millionen von Menschen leiden unter dieser Schlafstörung und nicht jeder kommt mit der Standardtherapie, der kontinuierlichen Überdruckbeatmung (CPAP), ausreichend gut zu Recht. Neben apparativen Alternativen bietet auch die Chirurgie zusätzliche Therapieoptionen an, die sich in den letzten Jahren deutlich weiterentwickelt haben.
Der 9. Jahreskongress der ISSS gastierte erstmals in Deutschland. Dabei tauschten sich internationale Experten über die neuesten Möglichkeiten in der Therapie und Diagnostik bei schlafbezogenen Atmungsstörungen - von der Neurostimulation bis hin zur Schnarchgeräuschanalyse via App vom 5. bis 7. April 2018 in München aus. Erstmals in der Geschichte des ISSS wurde der Kongress von einem Weiterbildungskursprogramm flankiert.

\section{I) Der Kongress wurde erstmals von einem Weiter- bildungskursprogramm flankiert}

Verschiedene neue chirurgische Verfahren am Zungenrund und am Weichgaumen waren zentraler Bestandteil des Kongresses. Beispielsweise sind unterschiedliche robotergestützte Resektionsverfahren am Zungengrund soweit weiterentwickelt worden, dass sie immer bessere klinische Ergebnisse liefern und ein wesentlicher Bestandteil des Therapiespektrums geworden sind. Auch die klassischen OP-Techniken am Weichgaumen in Form von Straffungen und Verlagerungsplastiken konnten in den letzten Jahren deutlich verfeinert werden und erzielen mittlerweile beachtliche Ergebnisse.

Highlight des Kongresses war die Keynote Lecture von Danny Eckert aus 


\section{Infobox 1}

Nächster ISSS-Kongress: 18.-20. Oktober

2020 Brisbane, Australien. https://www.

surgicalsleep2020.org/

Australien. Er beschäftigte sich in den letzten Jahren mit dem Thema der Phänotypisierung von Patienten mit OSA. Seit Jahren ist vielen schlafmedizinisch versierten Ärzten klar, dass die Ursache für eine OSA unterschiedlichen Ursprungs sein kann. Prof. Danny Eckert konnte anhand der Ursache der Schlafapnoe erstmalig eine Einteilung von Patienten in unterschiedliche Gruppen vornehmen. Seinen Übersichtsartikel finden Sie in diesem Heft. Somit wird in Zukunft wahrscheinlich eine zielgerichtete Therapie möglich sein, die es erlaubt, Patienten besser, weil individueller behandeln $\mathrm{zu}$ können.

\section{I) Anhand der Ursache der Schlafapnoe wurden erstmalig verschiedene Patientengruppen definiert}

Aber auch andere konservative Therapien wie das Schlafpositionstraining konnten in den letzten Jahren die Therapie der lageabhängigen OSA sinnvoll ergänzen. Die Analyse von Schlafatmungsgeräuschen mit künstlicher Intelligenz sind vielversprechende Ansätze, um eventuell in der Zukunft Atemaussetzer einfach per Smartphone zu detektieren und/oder Patienten mit OSA über längere Zeiträume zu Hause zu überwachen. Mithilfe der Sonographie konnten anatomische Besonderheiten evaluiert werden, die bei OSA-Patienten, nicht aber bei Schlafgesunden vorhanden sind.

Auch scheinen bereits Schlafmessverfahren (z. B. die Polysomnographie, PSG), wie sie heute in der klinischen Routine eingesetzt werden, eventuell ein zu hohes Bild der Rückenlage abzubilden und damit eventuell einen Effekt auf die Therapie der OSA zu haben.

Dieser Kongress war ein gelungener und sehr wichtiger Impulsgeber für die Diagnostik und Therapie schlafbezogener Atmungsstörungen. Eine Sammlung einiger dieser Highlights werden in diesem Heft publiziert. Wir hoffen, Ihnen mit diesen Artikeln eine Übersicht über die Vielfalt und die sich ständig weiterentwickelnden Felder der operativen Schlafmedizin geben zu können.

Als ehemalige Kongresspräsidenten wünschen wir Ihnen viel Spaß beim Lesen.

Clemens Heiser \& Thomas Verse

\section{Korrespondenzadresse}

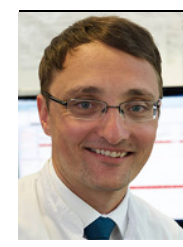

Prof. Dr. C. Heiser

Klinik und Poliklinik für Hals-, Nasen- und Ohrenheilkunde, Klinikum rechts der Isar, Technische Universität

München

Ismaninger Str. 22, 81675 München, Deutschland hno@heiser-online.com

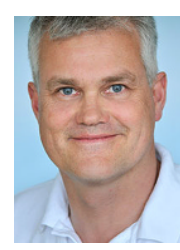

Prof. Dr. T. Verse

Klinik für Atemwegs-, Lungen- und Thoraxmedizin, Abteilung für HNO-Heilkunde, Kopf- und Halschirurgie, Asklepios Klinikum Harburg Eißendorfer Pferdeweg 52, 21075 Hamburg, Deutschland t.verse@asklepios.com

Interessenkonflikt. T. Verse gibt an, dass kein Interessenkonflikt besteht. C. Heiser ist als Berater für Inspire Medical Systems tätig. Er erhielt Reisekostenzuschüsse von Löwenstein Medical, Sutter Medizintechnik, Neuwirth Medical Products. Weiterhin ist er im Advisory Board von Galvani Bioelectronics.
Neue Leitlinie zu Husten bei Erwachsenen

Die Deutsche Gesellschaft für Pneumologie und Beatmungsmedizin weist in einer aktualisierten Leitlinie zur Diagnose und Therapie von Husten bei Erwachsenen darauf hin, dass die Ursache für chronischen Husten nicht immer in der Lunge oder den Atemwegen zu finden ist.

Akuter Husten ist meist Symptom einer Erkältung oder Grippe, bessert sich in der Regel nach zwei Wochen wieder und bedarf, außer bei schwerwiegenden Symptomen (Bluthusten, Atemnot, hohes Fieber) keiner weiteren Untersuchung. Ein über 8 Wochen oder länger andauernder Husten muss hingegen unter Einsatz von Lungenfunktionstests und Röntgenaufnahmen abgeklärt werden, um ernsthafte Erkrankungen wie einen Tumor oder eine COPD erkennen bzw. ausschließen zu können.

Auch muss eine Reizung der oberen Atemwege im Rahmen einer Entzündung der Nase oder Nasennebenhöhlen als Ursache von chronischem Husten in Betracht gezogen werden. Hier liegt ein überempfindlicher Hustenreflex zugrunde, bei dem bereits schwache Reize einen Husten auslösen. In derartigen Fällen ist die gemeinsame Diagnostik und Behandlung mit einem Hals-Nasen-Ohrenarzt sinnvoll.

Ebenfalls kann der aufsteigende Magensaft bei Sodbrennen bei überempfindlichem Hustenreflex chronischen Husten verursachen. Obwohl Sodbrennen gut medikamentös behandelt werden kann, bleibt der Hustenreflex häufig bestehen. Derzeit werden neue Medikamente entwickelt, die die Empfindlichkeit des Hustenreflexes über längere Zeit hemmen können. Klinische Tests hierzu dauern aber noch an.

Quelle:

P. Kardos et al.: Leitlinie der Deutschen Gesellschaft für Pneumologie und Beatmungsmedizin zur Diagnostik und Therapie von erwachsenen Patienten mit Husten. Pneumologie 2019; 73 (3); S.140-177 